\title{
Sex-specific chromatin states in mammalian fetal germ cells
}

\author{
Bluma J Lesch*, David C Page \\ From Epigenetics and Chromatin: Interactions and processes \\ Boston, MA, USA. 11-13 March 2013
}

\begin{abstract}
Background
Male and female mammalian germ cells follow identical developmental trajectories for the first half of embryogenesis, during which time they also maintain a pluripotentlike state. Beginning around day 13.5 of embryogenesis (E13.5), male and female germ cells initiate dramatically different developmental programs: female germ cells enter meiotic prophase, while male germ cells enter a $G_{0}$-like cell cycle arrest until after birth [1]. At this time, both male and female germ cells also lose the ability to establish pluripotent cell lines in culture [2]. As late as E12.5, male and female germ cells are morphologically identical, and few transcriptional differences can be detected $[1,3]$. To evaluate the coordination of sex-specific transcriptional states during this important interval in germ cell differentiation, we examined placement of the activating histone modification H3K4me3 and the repressive histone modification H3K27me3 in XX and XY murine germ cells before and during the initiation of sex differentiation.
\end{abstract}

\section{Materials and methods}

Germ cells expressing an Oct4-EGFP transgene [4] were isolated by flow cytometry from male and female mouse embryos at 12.5 or 13.5 days post coitus. Cells from multiple embryos were pooled and split into equal parts for ChIP against H3K4me3 or H3K27me3, followed by highthroughput sequencing. ChIP-seq data was compared to preexisting RNA-seq data from E12.5 or E14.5 male and female gonads.

\section{Results}

At day E12.5, at least $80 \%$ of H3K4me3 peaks associated with gene promoters were shared between male and female germ cells. Many genes marked by H3K4me3 in one sex but not the other at E12.5 were expressed specifically in germ cells of that sex one to two days later. Compared to H3K4me3, we found significantly more differences in placement of the H3K27me3 histone modification between males and females. Differences in H3K27me3 placement between sexes at E12.5 also corresponded to known differences in gene expression at later time points.

\section{Conclusions}

Mammalian germ cells display sex-specific chromatin configurations prior to the appearance of significant sex differences in morphology and gene expression. These differences in chromatin state anticipate sex differences in gene expression at later time points.

\section{Published: 18 March 2013}

\section{References}

1. McLaren A: Meiosis and differentiation of mouse germ cells. Symp Soc Exp Biol 1984, 38:7-23.

2. McLaren A: Primordial germ cells in the mouse. Developmental biology 2003, 262(1):1-15.

3. Jameson SA, Natarajan A, Cool J, DeFalco T, Maatouk DM, Mork L, Munger SC, Capel B: Temporal transcriptional profiling of somatic and germ cells reveals biased lineage priming of sexual fate in the fetal mouse gonad. PLoS Genet 2012, 8(3):e1002575.

4. Anderson R, Fassler R, Georges-Labouesse E, Hynes RO, Bader BL, Kreidberg JA, Schaible K, Heasman J, Wylie C: Mouse primordial germ cells lacking beta1 integrins enter the germline but fail to migrate normally to the gonads. Development 1999, 126(8):1655-1664.

doi:10.1186/1756-8935-6-S1-P45

Cite this article as: Lesch and Page: Sex-specific chromatin states in mammalian fetal germ cells. Epigenetics \& Chromatin 2013 6(Suppl 1):P45. 